\title{
A Priority Queue Model for Competition with Shared Spectrum
}

\author{
Chang Liu \\ EECS Department \\ Northwestern University, Evanston, IL 60208 \\ Email: changliu2012@u.northwestern.edu
}

\author{
Randall A. Berry \\ EECS Department \\ Northwestern University, Evanston, IL 60208 \\ Email: rberry@eecs.northwestern.edu
}

\begin{abstract}
Spectrum sharing has been put forward as a way to more efficiently use limited spectrum and thus increase wireless network capacity. This paper considers a scenario where a primary Service Provider (SP) shares spectrum with secondary SPs and competes for a common pool of customers. We study such a scenario using a model for price competition in which customers select a SP based on the sum of the SP's announced service price and the congestion incurred when using their service. Here, we assume that the primary has strict priority over the secondary and model the resulting congestion via a preemptive priority queue. We characterize the equilibrium of the resulting pricing game. In particular we find that when the service time has a small variance, secondary users can be excluded from the system, while the primary has to offer a lower price than it would if it were a monopolist due to the threat of entry. As the amount of available bandwidth increases, the primary SP's profit will decrease asymptotically to zero. In addition, for some scenarios, we show that social welfare may decrease with additional bandwidth and be less than that obtained without sharing.
\end{abstract}

\section{INTRODUCTION}

To meet the future demand of wireless data services, sharing has been proposed as an efficient way to unlock additional spectrum. For example, in the 2012 report of the President's Council of Advisors on Science and Technology (PCAST) [1], spectrum sharing is highlighted, especially for underutilized federal spectrum. Indeed, in the United States, spectrum sharing has been adopted in the TV white spaces [2] and the FCC is moving forward with proposals for sharing in the $3.5 \mathrm{GHz}$ band [3]. Sharing commercial bands between wireless service providers has also been suggested as an efficient way to increase network capacity, e.g., [4], [5].

There has been much research on the "primary-secondary" approach for spectrum sharing, where the secondary users can access a spectrum band provided they do not interfere with the primary users. This can be facilitated by sensing [6], a location-based approach [7] or a market-based approach [8]. In this paper, we focus on a primary-seocndary scenario where a wireless service provider (SP) is the primary spectrum user. This primary SP shares the spectrum band with a set of secondary SPs. Here, we consider a model of unlicensed secondary sharing as in the TV white spaces, meaning that any secondary SP can access the spectrum given

This research is supported in part by NSF grants CNS-1147786, SES1247984 and SES-1343381. they do not interfere with the primary. This can be contrasted with licensed secondary access in which a single SP has an exclusive license for secondary use [10]. Both licensed and unlicensed sharing are part of the 3-tier model proposed in the PCAST report [1] with tier 2 users having licensed secondary access and tier 3 users having unlicensed access. Our work can model a situation where the primary is such a tier 2 user and the secondaries are tier 3 users. Allowing such unlicensed sharing lowers the barrier for secondary SPs to enter the market and may enable them to provide low cost service; the competition among them and the primary can then potentially increase social welfare. Lowering the barrier of entry can also cause extra congestion for the secondaries and can affect the primary's pricing strategy. We present a model for studying such issues in this paper.

This paper continues a series of work in [9], [11], [12], where competition with spectrum sharing was studied. These works in turn were based models for competition with congestible resources studied in the operations and economics literature including [14], [15]. In these models, firms compete for customers by announcing prices. Customers select SPs based on the combination of their announced price and the amount of congestion in the resources used by the firm. In [14], [15], firms own seperate resources, while in the case of spectrum sharing certain resources are shared by SPs. Namely, in [?] a band of unlicensed spectrum is available for all SPs to use, in [11] licensed sharing of a band is considered, while in [9] unlicensed sharing of a band under the primary-secondary model is considered as in this paper. The main difference between this paper and the work in [9] is in how the congestion is modeled for the primary and secondary users. In [9], both the primary and secondary SP's congestion is given by a single function $g(x)$, that depends on the offered traffic $x$. The congestion of these firms then only differs in the offered traffic used: in the primary's case, this is simply the the number of users it serves, while for the secondaries it is the total number of users served by all SPs. Moreover, the main case studied in [9] was where this congestion function was a linear function of the traffic. In this paper, we consider at a more refined model based on a preemptive priority queue to model the congestion costs of the primary and secondary SPs. With this model, the secondary users' congestion is not given by the same function 


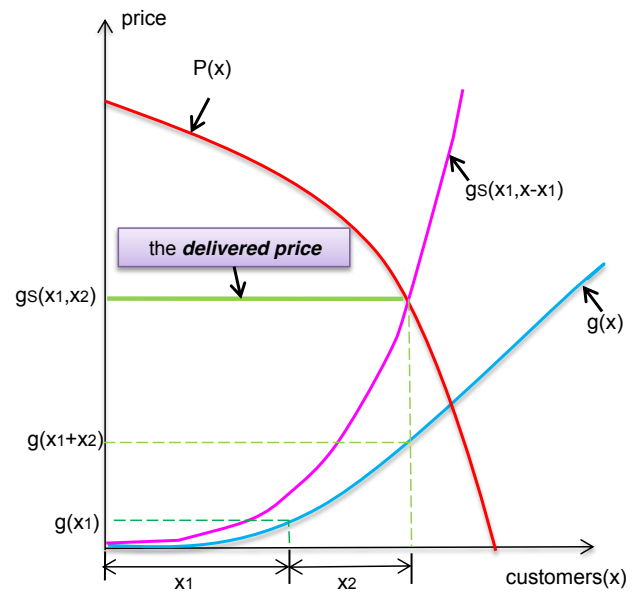

Fig. 1. Priority queue model for the pricing game with shared spectrum

of the total traffic as the primary, but secondary users incur an additional penalty due to the primary users that arrive while the secondary is waiting for service. Additionally, the congestion function is non-linear and depends on the variance of the service times as well as the average service rate. Our goal is understand what impact changing this congestion function has on the conclusions in [9].

Our main results show that this change in the congestion model leads to markedly different conclusions than those in [9]. In partiular, we show that while in [9] social welfare is always non-decreasing with the total bandwidth and no smaller than in a setting where shaing is not allowed, here social welfare can decrease with additional bandwidth and may be less than that obtained without sharing. This effect is similar to the work in [12] where competition between a licensed SP and SPs using a separate unlicensed band was studied and again social welfare was shown to decrease for a certain range of additional unlicensed bandwidth, though the actions leading to this decrease are different here. With this model we also show that the secondaries can be excluded from the market while the primary is forced to offer a lower price than it would as a monopolist due to the threat of entry. Again, such an outcome did not occur in [12]

The rest of the paper is organized as follows. Our model for price competition and congestion based on a priority queue are described in Section II. We analyze the competitive equilibrium and resulting welfare with and without spectrum sharing in Section III. Finally, we conclude in Section IV.

\section{Competition Model}

As in [9], we consider a competition model for a wireless service market where SPs announce prices for their service to compete for a common pool of customers. The service quality offered by each SP is modeled by a congestion cost. In this paper, we model the congestion as the average delay for customers to receive service assuming that customers are served sequentially by SPs using the entire spectrum in a given area. Each customer then chooses one SP based on all the SPs' offered services and prices. Specifically, customers select the SP that has smallest delivered price, which is the sum of their announced price and congestion cost.

\section{A. Supply and Demand}

Supply: We focus on a wireless service market where there is one incumbent SP. Without spectrum sharing, it acts as a monopolist. With spectrum sharing, it announces a price, $p$ and competes simultaneously with $N>2$ secondary SPs who announce prices, $\mathbf{p}^{\mathbf{S}}=\left(p_{1}^{S}, p_{2}^{S}, \ldots, p_{N}^{S}\right)$ and serve $\mathbf{X}=$ $\left(x_{1}^{S}, x_{2}^{S}, \ldots, x_{N}^{S}\right)$ number of customers, respectively.

The primary's service quality is given by its congestion cost $g(x)$, which only depends on $x$, the number of customers (primary users) it is serving. This characterizes a scenario where the primary users do not experience any interference from the secondary users. On the other hand, the secondaries' service is degraded compared to the primary as they encounter longer delays due to giving priority to the primary and waiting for available spectrum to be released. Their congestion is given by $g_{S}\left(x_{1}, x_{2}\right)$, where $x_{2}=\sum x_{i}^{S}$ is the total number of secondary users. This characterizes the fact that the secondary users not only receive interference from themselves but also the primary users. Generally, we assume that $g($.$) and g_{S}($.$) are continuous, increasing and$ convex functions with $g(0)=g_{S}(0)=0$ (see Fig. 1); the specific functions we consider in this paper will be defined in Section II.B.

Demand: We assume a single mass of infinitesimal customers and normalize the total customer mass to be 1 . We use an inverse demand function $P(x)$ to characterize the customers' demand for service at a certain delivered price. This is a non-increasing, left-continuous, concave function that indicates the delivered price at which a mass of $x$ customers are willing to pay for service. As noted before, the delivered price is the sum of the price each customer needs to pay and the congestion they experience. Each customer can choose to be a primary user or a secondary user giving them the delivered prices $p_{1}+g\left(x_{1}\right)$, or $p_{k}^{S}+g_{S}\left(x_{1}, x_{2}\right)$, respectively. Otherwise, if the equilibrium delivered price is higher than what a customer is willing to pay, she will not receive service. We additionally assume that $P(x)>0$ for small enough value of $x$ so that there always exist a small enough price at which some customers will be willing to pay for service. Each customer seeks to choose an SP who offers the lowest delivered price (provided the price is no greater than what they are willing to pay). If more than one service provider has the same delivered price, a customer chooses one of the low price SPs uniformly at random. Thus, given a set of prices, $\left(p, \mathbf{p}^{S}\right)$, the customers receiving service must be in a Wardrop equilibrium [17], meaning that:

1) The delivered price for all active SPs (i.e., those serving customers) are equal and strictly less than the delivered price of any non-active SP;

2) If the active SPs are serving a total of $x=x_{1}+x_{2}$ customers, then the delivered price of these SPs, $y$ satisfies $y \leq P(x)$ and $y>P(x+\epsilon)$ for any $\epsilon>0$. 
Note the second condition specifies that any customer not being served sees a delivered price higher than it is willing to pay. Note if $P(x)$ is continuous at $x$, then this is equivalent to having $y=P(x)$. In the next section, for analysis, we assume that customers are homogeneous in their demand, i.e., all customers are willing to pay up to the same delivered price, in which case $P(q)$ has a "box" shape as seen in Fig. 2.

\section{B. Congestion: Priority Queue}

As stated earlier we consider a scenario where all customers are served sequentially with the primary SP's customers having priority over the secondary SP's customers. To model the congestion function for both the primary and secondary SPs, we adopt a model based on the expected average waiting time in a M/G/1 system with two priority classes: class 1 and class 2. Class 1, the primary, has the higher (preemptive) priority, modeling a case where the secondary instantly stops serving customers when the primary needs to use the spectrum. ${ }^{1}$ For such a system, the average waiting times $W_{1}$ and $W_{2}$ for each class $i$, respectively, are [18]:

$$
\begin{array}{ll}
\text { Primary: } & W_{1}=\frac{\frac{1}{2} \lambda_{1} \bar{Y}_{1}^{2}}{1-\frac{\lambda_{1}}{\mu_{1}}}, \\
\text { Secondary: } \quad W_{2}=\frac{\frac{1}{2}\left(\lambda_{1} \bar{Y}_{1}^{2}+\lambda_{2} \bar{Y}_{2}^{2}\right)}{\left(1-\frac{\lambda_{1}}{\mu_{1}}\right)\left(1-\frac{\lambda_{1}}{\mu_{1}}-\frac{\lambda_{2}}{\mu_{2}}\right)} .
\end{array}
$$

where $\lambda_{i}, \bar{Y}_{i}=1 / \mu_{i}$, and $\bar{Y}_{i}^{2}$ are respectively the arrival rate and the first two moments of the service time of class $i$. We will use these as the congestion costs for primary and secondary services.

To simplify notations, we let $a_{i}=\bar{Y}_{i}^{2} / 2, b_{i}=\bar{Y}_{i}=1 / \mu_{i}$ for each class $i$, and further equate the arrival rates with the number of customers served by the primary and secondary SPs, i.e., $x_{1}=\lambda_{1}$, and $x_{2}=\lambda_{2}$. We then specify the congestion functions as follows:

$$
\begin{array}{ll}
\text { Primary: } & g\left(x_{1}\right)=\frac{a_{1} x_{1}}{1-b_{1} x_{1}}, \\
\text { Secondary: } & g_{S}\left(x_{1}, x_{2}\right)=\frac{a_{1} x_{1}+a_{2} x_{2}}{\left(1-b_{1} x_{1}\right)\left(1-b_{1} x_{1}-b_{2} x_{2}\right)} .
\end{array}
$$

Also for most of our analysis, we assume that $a_{1}=a_{2}$ and $b_{1}=b_{2}$, then we have

$$
\begin{array}{r}
g_{S}\left(x_{1}, x_{2}\right)=\frac{a_{1}\left(x_{1}+x_{2}\right)}{\left(1-b_{1} x_{1}\right)\left[1-b_{1}\left(x_{1}+x_{2}\right)\right]} \\
=g\left(x_{1}+x_{2}\right) \frac{1}{\left(1-b_{1} x_{1}\right)},
\end{array}
$$

i.e., the congestion seen by the secondary users is given by the same congestion function $g$ as used for the primary users, but evaluated with the total traffic $x_{1}+x_{2}$, and further increased by the multiplicative term $1 /\left(1-b_{1} x_{1}\right)$, which depends on the primary's traffic.

\footnotetext{
${ }^{1}$ In practice of course there will be some delay before the secondary releases the channel to the primary; we ignore this here.
}

Note that since $a_{1}$ and $b_{1}$ are proportional to the second and first moment of the service time, $Y_{1}$, it follows that $2 a_{1}=$ $b_{1}^{2}+\sigma_{Y}^{2}$, where $\sigma_{Y}^{2}$ is the variance of the service time, so that that it must be that $a_{1} \geq b_{1}^{2} / 2$, with equality when $\sigma_{Y}^{2}=0$.

\section{Equilibrium}

Given the demand and congestion functions defined in the previous section, we define a (pure strategy) Nash equilibrium of the overall pricing game to be a set of prices $\left(p, \mathbf{p}^{S}\right)$ and demands $\left(x_{1}, \mathbf{X}\right)$, which satisfy the Wardrop equilibrium conditions as above with the additional property that no SP can increase its profit by unilaterally changing its price.

Given such an equilibrium, the consumer welfare, $S_{c}$ is defined by the sum of each customer's welfare, which is the price she values the service, $P(x)$, minus the delivered price she pays. This is equivalent to the area bounded by the curve of $P(x)$ and the delivered price (see Fig. 1). The firm profit, $f$ is given by the sum of the profits made by all SPs, which is the product of the customers they serve and their announced price. The social welfare, $S$, of the entire economy is the sum of the firm profit and the consumer welfare.

\section{Monopoly}

Without spectrum sharing, the primary SP acts as a monopolist. It announces the price to maximize its own profit given the market demand. The optimal price is given by solving the following optimization problem:

$$
\begin{array}{ll}
\max _{p, x} & p x \\
\text { subject to } & p+g(x) \leq P(x), \\
& 0 \leq x \leq 1
\end{array}
$$

Since the objective is continuous and the constraint set is compact and non-empty, this problem must have a solution. Here, the first constraint ensures that the number of customers served satisfies the second Wardrop equilibrium condition. If $P(x)$ is continuous with $P(1)=0$, then this constraint will be met with equality for any choice of $P$; if this is not the case, then at optimality this constraint must still be tight at optimality, since if it was not tight, the monopolist could increase the price and get more revenue. Hence, we can replace the first constraint with $p+g(x)=P(x)$ and further solve for $p$ in terms of $x$. This enables us to write the problem as an optimization only over $x$ with the objective $x(P(x)-g(x))$ subject to $0 \leq x \leq 1$. As shown in the appendix, this objective is strictly concave over the constraint set and so the problem will have a unique solution.

\section{E. Unlicensed Spectrum Sharing}

With unlicensed spectrum sharing, we assume that there are multiple secondary SPs in the market. Since each secondary SP offers a service with the same congestion cost $g_{s}\left(x_{1}, x_{2}\right)$, the provider with the lowest announced price will capture the entire market of secondary users. This drives the secondary SPs' announced prices to zero, as stated in 


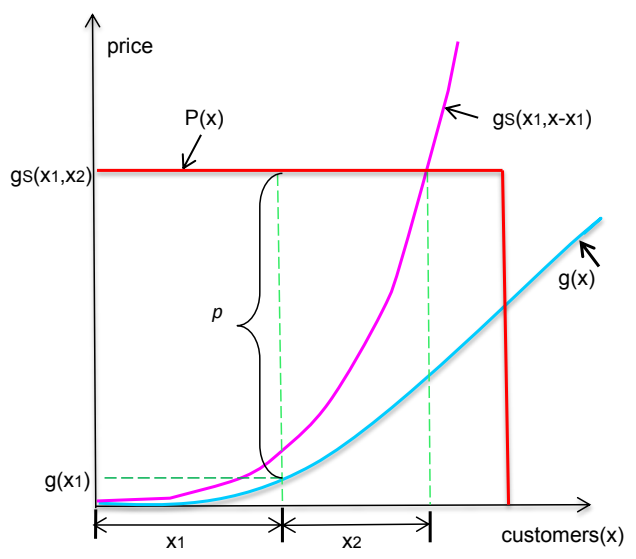

Fig. 2. An example of the pricing game with shared spectrum when bandwidth is limited.

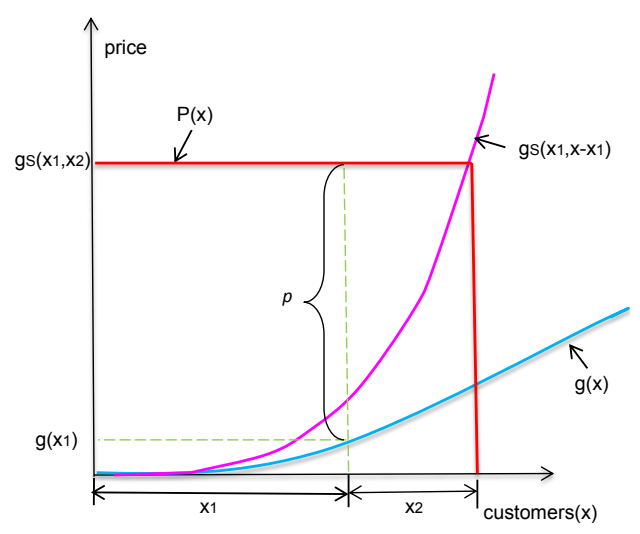

Fig. 3. An example of the pricing game with shared spectrum when bandwidth is increased but the delivered price stays at the price cap.

the following result, which can be derived using a similar argument as in [9].

Lemma 2.1: With unlicensed shared spectrum and $N \geq 2$ secondary SPs, in any Nash equilibrium, all active secondary SPs will charge zero price to customers, i.e., $p_{i}^{S}=0$ for all $i$.

Recall, an active SP refers to one that is serving customers. Notice that since each secondary SP's announced price is zero, their profit will also be zero. Hence, any SP will be indifferent from being active and being inactive and announcing a larger price than zero. However, to get a Nash equilibrium for the overall game in such cases will require that at least one SP announces a price of zero. Also note that we did not include any marginal cost $c$ for the secondaries to deliver service; if we did include this, the equilibrium price for any active secondary SP would be equal to $c$.

With at least two secondary SPs, this lemma shows that the announced price of all active secondaries will be zero, and so if the secondaries are serving any customers in an equilibrium, then their delivered price will be equal to their congestion $g_{S}\left(x_{1}, x_{2}\right)$. It follows from the Wardrop equilibrium equilibrium conditions that given that the primary SP is serving $x_{1}$ customers, the total number of customers served in the entire market in equilibrium, $x^{*}$, must be the solution of the following optimization problem:

$$
\begin{array}{ll}
\max & x \\
\text { subject to: } & g(x) \frac{1}{1-b_{1} x_{1}} \leq P(x), \\
& x_{1} \leq x \leq 1
\end{array}
$$

It can be seen that this problem will have a solution if and only if $g\left(x_{1}\right) \frac{1}{1-b_{1} x_{1}} \leq P\left(x_{1}\right)$. In the case were a solution does not exist, then there can not be any active secondary SPs. If a solution does exist, then it can also be seen that it will be unique. In particular, if $P(x)$ is continuous at $x^{*}$, then at the optimal solution the first constraint must be tight, i.e., the solution is given by solving

$$
g(x) \frac{1}{1-b_{1} x_{1}}=P(x) .
$$

Let $x_{1, m}$ be the number of customers the primary would serve when it was a monopolist. Then, considering (2) for this value of $x_{1}$, it follows that if (2) has no solution, the primary will again serve $x_{1, m}$ customers with unlicensed sharing and no secondaries will be active. Otherwise, if (2) has a solution for $x_{1}=x_{1, m}$, then there are two possibilities: (i) the delivered price $g\left(x^{*}\right) \frac{1}{1-b_{1} x_{1, m}}$ equals that obtained by a monopolist or (ii) this delivered price is smaller than that under a monopolist. In the first case the primary can continue serving $x_{1, m}$ customers at the monopoly price and any additional customers are served by the secondary SPs. In the second case, the primary SP can no longer obtain the same revenue as a monopolist. Indeed, it follows that in any equilibrium $g\left(x^{*}\right) \frac{1}{1-b_{1} x_{1}}$ will be a cap on the delivered price and so the primary must either announce a lower price or serve fewer customers. Note also that $x^{*}$ and thus this cap on the delivered price depends on the number of customers the primary SP is serving $\left(x_{1}\right)$; namely, as the primary SP serves fewer customers, $x^{*}$ will be non-decreasing and so the price cap will be non-increasing. To compare with the work in [9], there sharing also resulted in a cap on the delivered price, but that cap did not depend on the primary's traffic, $x_{1}$. This dependency provides a new potential strategic option for the primary since increasing the number of customers it is serving can cause more congestion for the secondaries and increase the delivered price cap. In the following sections, we will see that with a large variance of the service rate, the primary will exploit this option and may even exclude the secondaries from the market.

We now turn to studying the Nash equilibria of the overall pricing game with shared spectrum. Under the assumed properties for the inverse demand, the next theorem shows that a Nash Equilibrium always exists.

Theorem 2.2: For the priority queue model with shared spectrum a Nash equilibrium always exists.

Proof: If (??) has no solution when the primary serves $x_{1, m}$ customers, then the existence of a Nash equilibrium is immediate as the primary can simply acts as a monopolist and 


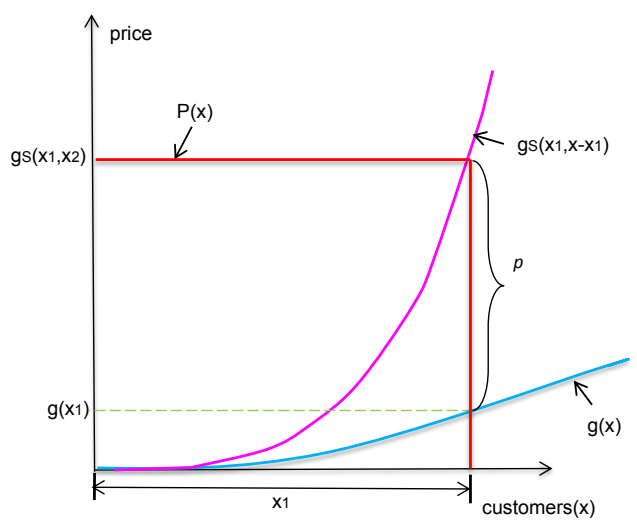

Fig. 4. An example of pricing game with shared spectrum when the primary is serving the entire market with a non-monopoly price

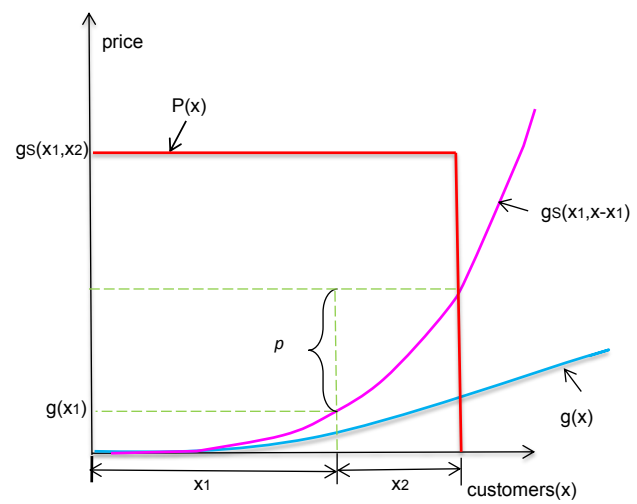

Fig. 5. An example of pricing game with shared spectrum when the bandwidth is large enough for secondaries to enter and the delivered price is below the cap.

no secondary will enter the market. Otherwise, the primary must account for the "price cap" imposed by the secondary and so is faced with the problem

$$
\begin{array}{ll}
\max & p x_{1} \\
\text { subject to: } & p+g\left(x_{1}\right)=g(x) \frac{1}{1-b_{1} x_{1}}, \\
& g(x) \frac{1}{1-b_{1} x_{1}} \leq P(x), \\
& p \geq 0, x_{1} \leq x \leq 1 .
\end{array}
$$

Again this problem has a continuous objective and a compact constraint set and so must have a solution. If the primary SP announces the price $p^{*}$, given by solving this and all secondary SPs announce a price of zero, then this must be a Nash equilibrium.

\section{COMPETITION AND Welfare ANALYSiS}

In this section, we assume that the inverse customer demand function is homogeneous, i.e., it has a "box" shape. All the customers in the market are willing to pay up to the same level of price, which we also normalize to be one, so

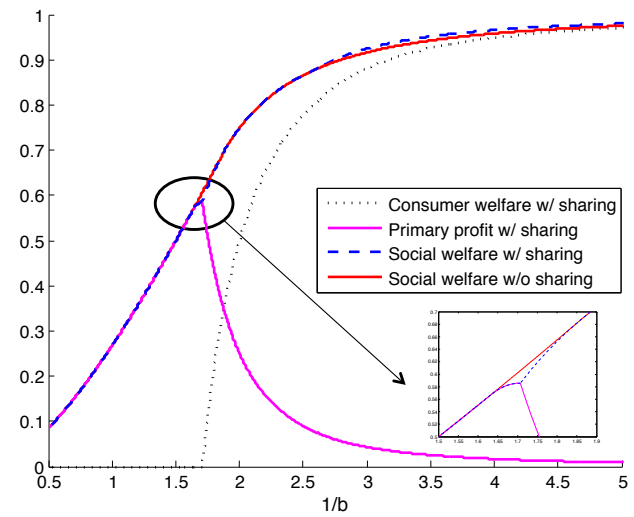

Fig. 6. Welfares of the primary and secondary pricing game with and without shared spectrum with $\sigma_{Y}^{2}=0$.

that

$$
P(x)= \begin{cases}1, & 0 \leq x \leq 1 \\ 0, & \text { otherwise }\end{cases}
$$

\section{A. Without Spectrum Sharing}

Without sharing spectrum, we can re-write the primary SP's optimization in (1) as

$$
\begin{array}{ll}
\max & \left(1-\frac{a_{1} x}{1-b_{1} x}\right) x \\
\text { subject to } & 0 \leq x \leq 1,
\end{array}
$$

where as discussed in Section II-D we have used the fact that the first constraint in (1) holds with equality. Solving this, we have that without spectrum sharing the primary will serve

$$
x^{*}=\min \left(\frac{1}{b_{1}}-\sqrt{\frac{1}{b_{1}}-\frac{1}{b_{1}\left(a_{1}+b_{1}\right)}}, 1\right)
$$

customers.

As $b_{1}$ is the inverse of average service rate, we will view $1 / b_{1}$ as the bandwidth available for the primary. The next lemma characterizes how the primary's profit varies with this amount of bandwidth.

Lemma 3.1: Without spectrum sharing, the primary SP's profit is non-decreasing in the bandwidth $1 / b_{1}$.

This lemma follows from noting that the profit of the monopolist is given by

$$
\pi_{M}=x^{*} \times \max \left(\left(1-\frac{a_{1} x^{*}}{1-b_{1} x^{*}}\right),\left(1-\frac{a_{1}}{1-b_{1}}\right)\right)
$$

which in increasing in $1 / b_{1}$ for $a_{1} \geq b_{1}^{2} / 2$, which as noted earlier must be true.

In the monopoly case with homogeneous demand it is easy to see that consumers will not obtain any welfare, i.e., the delivered price will be equal to 1 . Hence in this case, the primary's profit is equal to the overall social welfare, a plot of this as a function of the available bandwidth is shown in Fig. 6 and Fig. 9 when the variance of $\bar{Y}$ is 0 and 1, respectively. 


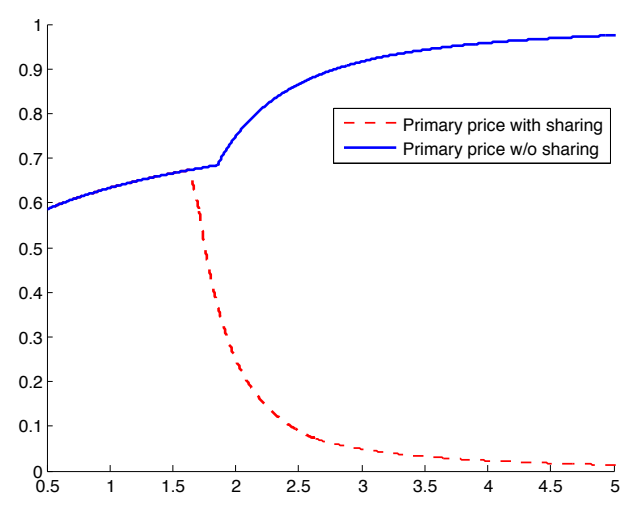

Fig. 7. Primary price with and without shared spectrum $\left(\sigma_{Y}^{2}=0\right)$.

\section{B. Spectrum Sharing}

With shared spectrum, the primary SP must compete with the secondary SPs. From the preliminary results in Sect. II, the secondaries will charge zero price to their customers, which indicates that if any secondaries are active then the delivered price is just their congestion $g_{S}\left(x_{1}, x_{2}\right)=g\left(x_{1}+\right.$ $\left.x_{2}\right) \times \frac{1}{1-b_{1} x_{1}}$. Further we know that when (2) has no solution for $x_{1}$ equal to the value in (4), then no secondary will be active. For the remainder of this section we turn to the case were this is not true. In such cases, the primary SP's profit maximization is given by solving:

$$
\begin{array}{ll}
\max & p x_{1} \\
\text { subject to } & \frac{a_{1}(x)}{1-b_{1}(x)} \frac{1}{1-b_{1} x_{1}} \leq 1, \\
& p+\frac{a_{1} x_{1}}{1-b_{1} x_{1}}=\frac{a_{1}(x)}{1-b_{1}(x)} \frac{1}{1-b_{1} x_{1}} \\
& 0 \leq x_{1} \leq x \leq 1 .
\end{array}
$$

As the inverse demand $P(x)$ has a "box" shape, it is convenient to separate the solution to this problem into four regions shown in Fig. 2 - Fig. 5. The first region, as in Fig. 2, is when the bandwidth is limited so that not all customers are served. In this case, the primary is serving part of the customers and the secondary users have occasional access to the spectrum but the delivered price remains at the ceiling price of 1 . In this case, enabling secondary sharing does not affect the primary's profit as indicated in Fig. 6 and Fig. 8. As the bandwidth increases, the primary and secondary will serve more customers until all of the customers in the market are being served, which is the second region, as in Fig. 3. At this time, the primary is serving $x_{1}=\frac{1-a_{1}-b_{1}}{b_{1}\left(1-b_{1}\right)}$ customers. Here, if the primary wants to maintain the delivered price at 1 , it has to decrease its announced price to gain more customers while causing more congestion for the secondaries. For a range of bandwidth, this is the optimal action of the primary. As shown in Fig. 8, the primary is now serving more customers than in the monopoly case. This market expansion also reduces the number of secondary users which, as shown in Fig. 8, decreases to zero for some range of bandwidth.

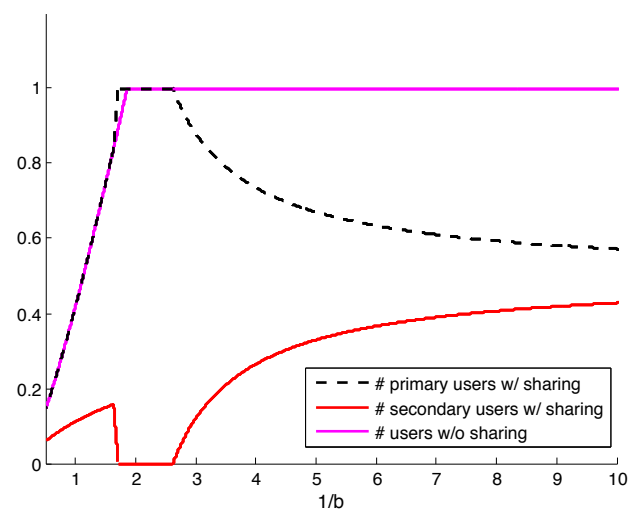

Fig. 8. Number of primary and secondary users with and without shared spectrum $\left(\sigma_{Y}^{2}=0\right)$.

In this case, the primary is serving the entire market while announcing a non-monopoly price due to the threat of entry as shown in Fig. 7 and Fig. 4. This price cap due to the threat of entry may not always exists as shown in another example in Fig. 9 and Fig. 10 where the variance of $Y$ is large. In that case, the primary will instead serve fewer customers to gain more profit. The condition for the primary to serve the entire market with a non-monopoly price is given by the following theorem.

Theorem 3.2: If and only if

$$
a_{1} \leq\left(b_{1}-1\right)^{2},
$$

then if the primary SP serves the entire market it must offer a price lower than the monopoly price due to the threat of entry from the secondaries.

Proof: First consider the monopoly case. In that case it follows that the primary will serve the entire market when it announces a price of

$$
p_{1}=1-\frac{a_{1}}{1-b_{1}},
$$

which results in a delivered price of $p_{1}+g(1)=1$. Furthermore, from the analysis of the monopoly case, we know that with sufficient bandwidth such a price will be optimal. For this price to be an equilibrium in the case of shared spectrum it must be that no secondary SP is active and imposing a lower price cap than 1 on the primaries delivered price. This is equivalent to requiring that the delivered price of each secondary SP to be no less than 1, when there is no secondary traffic, i.e. that

$$
\begin{aligned}
1 & \leq g_{S}(1,0) \\
& =\frac{a_{1}}{\left(1-b_{1}\right)^{2}} .
\end{aligned}
$$

In other words, we have shown that if $a_{1} \geq\left(1-b_{1}\right)^{2}$, then the primary will be able to serve the entire market and still charge the monopoly price. On the other hand, if this condition is not true, then the only way the primary can serve the entire market is by charging a price lower than the monopoly price. 


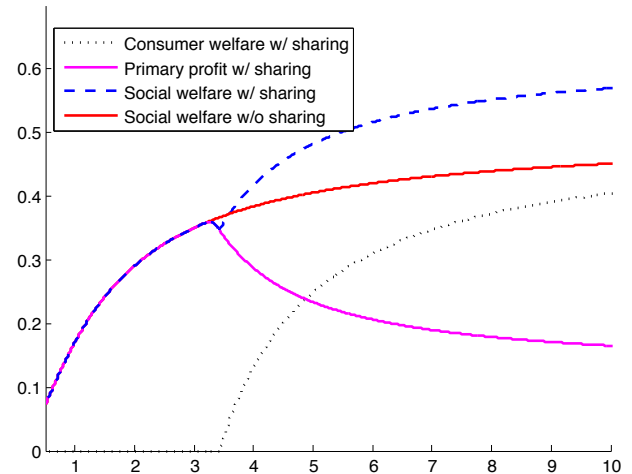

Fig. 9. Welfares of the primary and secondary pricing game with and without shared spectrum $\left(\sigma_{Y}^{2}=1\right)$.

As the bandwidth becomes larger, it will eventually not be optimal for the primary to decrease it price so as to gain more customers from the secondaries. After that point, the primary will serve fewer customers and the number of customers served by the secondaries will increase. From Fig. 8, it can be seen that with large enough bandwidth, the primary and secondary SPs will divide the market, which provides more consumer welfare and social welfare compared with monopoly case. It follows that the primary is losing profit as given in the following theorem.

Theorem 3.3: With large enough bandwidth available, the primary SP gains less profit as bandwidth increases

This is also shown in Fig. 6 and Fig. 9. Unlike the monopoly case, the primary profit shrinks asymptotically to zero as the amount of bandwidth increases. Also as shown in Fig. 4, as the primary decreases the number of customers it is serving, this causes more congestion in the shared band and its profit slightly decreases. This also decreases social welfare. This happens more dramatically in the case where the variance of the service time is larger as shown in Fig. 9. There it is shown that for a range of bandwidth, social welfare decreases and is below that obtained by a monopolist. This is in contrast to our previous model in [9] and is summarized in the following theorem.

Theorem 3.4: Spectrum sharing gives more social welfare than no sharing with large enough bandwidth. However, It may decrease social welfare for some range of bandwidth.

As seen from Fig. 6 and Fig. 9, spectrum sharing outperforms the monopoly case in terms of social welfare when the bandwidth is large. The drop in social welfare for some range of bandwidth is due to the fact that the primary has an incentive to serve more customers to cause congestion in the shared band and increase the delivered price cap as discussed previously.

\section{CONCLUSions}

In this paper, we considered a scenario where a primary service providers shares its spectrum with multiple secondary service providers using a preemptive priority rule. Price

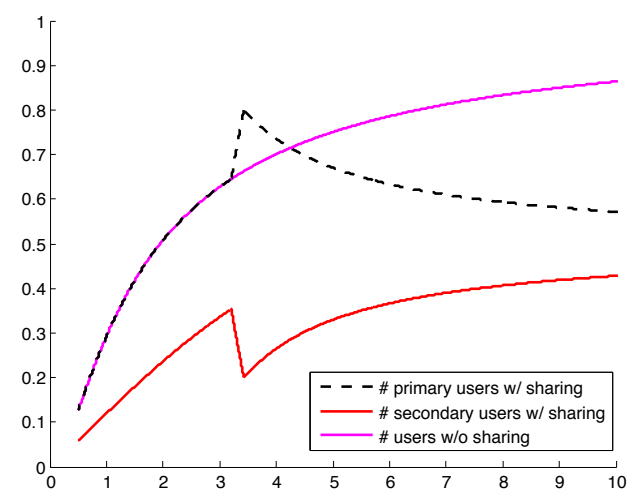

Fig. 10. Number of primary and secondary users with and without shared spectrum $\left(\sigma_{Y}^{2}=1\right)$.

competition among the primary and secondary for a common pool of customers was studied. Here, It was shown that the social welfare can decrease when the amount of bandwidth for sharing is increased due to the market expansion strategy of the primary. Also with spectrum sharing, it was shown that the primary may serve the entire market with a nonmonopoly price due to the potential competition from the secondaries. With greater variance of the service time, the drop in social welfare happens at a larger bandwidth.

In most of our analysis, we assumed the primary and secondary have the same first and second moments of service times, i.e., $a_{1}=a_{2}$ and $b_{1}=b_{2}$. We briefly comment on the case where these are different. If either $a_{2}$ or $b_{2}$ are increased (i.e. the secondaries have a slower service rate or larger variance), then this will cause the secondaries to have higher congestion and thus enable the primary to sustain monopoly profits over a larger range of bandwidths $1 / b_{1}$. Next suppose we consider making the secondary service times smaller by requiring them to send smaller packets (but at a proportionally larger arrival rate. For example, we could split the packet sizes in half abut double the arrival rates, i.e., setting $b_{2}^{\prime}=b_{2} / 2$ and $\lambda^{\prime}=2 \lambda$, additionally if the packets are fixed size so that $a_{2}=b_{2}^{2} / 2$, then after splitting the packets, $a_{2}$ will be scaled to $a_{2} / 4$. It can be seen that such a change will reduce the congestion of the secondary (intuitively this is because smaller packets will incur fewer interruptions from the the primary). Such a change will improve the secondaries performance, but may reduce the primary firm's profits. An example of how the primary firm's profits varies as the service time and variance of the secondary users traffic is varied is shown in Fig. 11. From this it can be seen that the primary firm's profit decreases with the average service time for secondary users, $b_{2}$ and increases with second moment of the service time.

In addition, general demand functions can be explored in future works as can models that include the investment decisions of the service providers. 


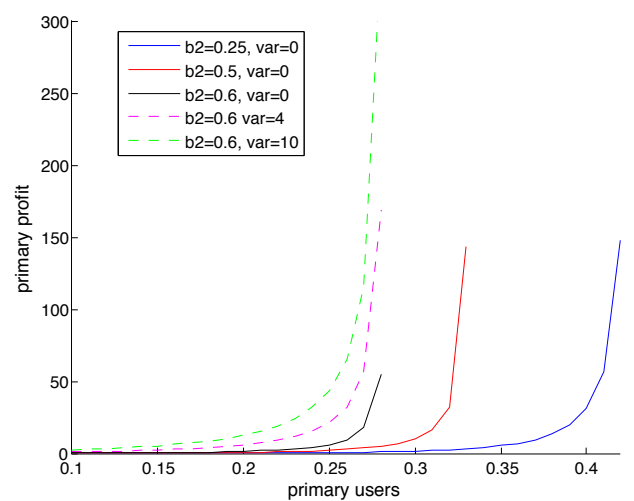

Fig. 11. Effect on the primary firm profit due to different average secondary services time, $b 2$, and different variance of the secondary service time.

\section{REFERENCES}

[1] PCAST, Realizing the Full Potential of Government-Held Spectum to Spur Economic Growth. President's Council of Advisors on Science and Technology, July 2012.

[2] Federal Communications Commission, "Unlicensed operation in the TV broadcast bands ; additional spectrum for unlicensed devices below 900 $\mathrm{MHz}$ and in the $3 \mathrm{GHz}$ band" second memorandum opinion and order, 2010.

[3] Federal Communications Commission, "Amendment of the commission's rules with regard to commercial operations in the 3550-3650 MHz band," docket no. 12- 354, Notice of Proposed Rulemaking, Dec. 2012.

[4] S. Panichpapiboon, and J.M. Peha, "Providing secondary access in a cellular network, Int. Conf. Wireless Networks, pp. 591-7, June 2003.

[5] R. Saruthirathanaworakun and J.M. Peha, "Dynamic Primary-Secondary Spectrum Sharing with Cellular Systems,"IEEE Crowncom 2010.

[6] Cabric, D., S. Mishra, and R. Brodersen, "Implementation issues in spectrum sensing for cognitive radios," 2004 IEEE Asilomar Conference on Signals, systems and computers, 2004.

[7] D. Gurney, G. Buchwald, L. Ecklund, S.L. Kuffner, J. Grosspietsch, "Geo-Location Database Techniques for Incumbent Protection in the TV White Space", IEEE DySPAN 2008.

[8] J. Huang, R. Berry, and M. Honig, "Auction-based spectrum sharing," ACM Mobile Networks and Applications, vol. 11, no. 3, pp. 405-418, June 2006.

[9] C. Liu and R. Berry, "Competition with Shared Spectrum," 2014 IEEE DySPAN, Apr. 2014.

[10] R. Berry, M. Honig, V. Subramanian, T. Nguyen, R. Vohra, "Market Structures for Wireless Services with Shared Spectrum," Allerton Conference, 2013.

[11] C. Liu, R. A. Berry, "Competition with Licensed Shared Spectrum", Proceedings of 48th Annual Conference on Information Sciences and Systems, Mar. 2014.

[12] T. Nguyen, H. Zhou. R. Berry, M. Honig, and R. Vohra, "The impact of additional unlicensed spectrum on wireless services competition," 2012 IEEE DySPAN, pp. 146-155, May 2011.

[13] H. Zhou, R. Berry, M. Honig, R. Vohra, "Investment and Competition in Unlicensed Spectrum", 46th Annual Conference on Information Sciences and Systems (CISS), 2012.

[14] D. Acemoglu, A. Ozdaglar, "Competition and Efficiency in Congested Markets," Mathematics of Operations Research., Feb. 2007

[15] R. Johari, G. Y. Weintraub, B. V. Roy,"Investment and Market Structure in Industries with Congestion," Operations Research., Sep. 2010

[16] R. Berry, M. Honig,T. Nguyen, V. Subramanian, H. Zhou, R. Vohra, "On the nature of Revenue-Sharing Contracts to Incentivize SpectrumSharing", IEEE INFOCOM, 2013.

[17] J. G. Wardrop, "Some theoretical aspects of road traffic research," Proceedings, Institute of Civil Engineers, PART II, vol. 1, pp. 325378, 1952.

[18] D Bertsekas, R Gallager, "Data Networks," Prentice Hall, 1992.

\section{APPENDIX}

In this appendix we give a short justification as to why the objective $x(P(x)-g(x))$ is strictly concave. Note that under our assumptions $f(x)=P(x)-g(x)$ is a concave and strictly decreasing function on $[0,1]$ (but not necessarily continuous or differentiable). Let $L(x)=x f(x)$. To see that $L(x)$ is strictly concave in $x$, we need for all $x$ and $y$ and $\lambda$ in $[0,1]$

$$
L(\lambda x+(1-\lambda) y)>\lambda L(x)+(1-\lambda) L(y)
$$

i.e.,

$(\lambda x+(1-\lambda) y) f(\lambda x+(1-\lambda) y)>\lambda x f(x)+(1-\lambda) y f(y)$.

Since $f(x)$ is concave, then

$$
\begin{aligned}
& (\lambda x+(1-\lambda) y) f(\lambda x+(1-\lambda) y) \\
\geq & (\lambda x+(1-\lambda) y)(\lambda f(x)+(q-\lambda) f(y)) \\
= & \lambda(\lambda x+(1-\lambda) y) f(x)+(1-\lambda)(\lambda x+(1-\lambda) y) f(y) \\
= & \lambda(1+\lambda-1) f(x)+\lambda(\lambda) y f(x)+(1-\lambda) y f(y) \\
& -\lambda(1-\lambda) y f(y)+(1-\lambda) \lambda x f(y) \\
= & \lambda x f(x)+(1-\lambda) y f(y)+(\lambda-1) \lambda x f(x) \\
& +\lambda(1-\lambda) y f(x)-\lambda(1-\lambda) y f(y)+(1-\lambda) \lambda x f(y) \\
= & \lambda x f(x)+(1-\lambda) y f(y)+\lambda(1-\lambda)(y-x)(f(x)-f(y)) .
\end{aligned}
$$

As $f(x)$ is strictly decreasing, the underlined term is strictly positive. Thus we complete our proof that $(\lambda x+(1-$ ג)y) $f(\lambda x+(1-\lambda) y)>\lambda x f(x)+(1-\lambda) y f(y)$. 